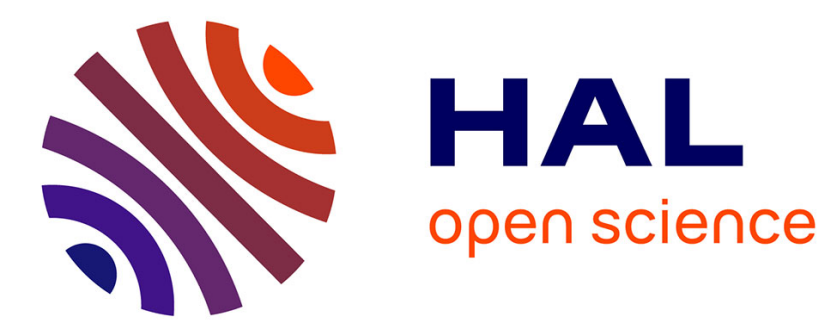

\title{
Ce qui songe au fond de l'image
}

Jean-Michel Durafour

\section{To cite this version:}

Jean-Michel Durafour. Ce qui songe au fond de l'image: Rêve, rêverie, film, autour de Jean Renoir. Ligeia, dossiers sur l'art, 2014, Patricia-Laure Thivat (dir.), Le rêve au cinéma, 129-132, pp.67-79. hal-01368834

\section{HAL Id: hal-01368834 https://hal.science/hal-01368834}

Submitted on 20 Sep 2016

HAL is a multi-disciplinary open access archive for the deposit and dissemination of scientific research documents, whether they are published or not. The documents may come from teaching and research institutions in France or abroad, or from public or private research centers.
L'archive ouverte pluridisciplinaire HAL, est destinée au dépôt et à la diffusion de documents scientifiques de niveau recherche, publiés ou non, émanant des établissements d'enseignement et de recherche français ou étrangers, des laboratoires publics ou privés. 


\title{
Ce qui rêvasse au fond de l'image. Rêve, rêverie, film, autour de Jean Renoir
}

\author{
Jean-Michel Durafour
}

\section{paru initialement dans Ligeia, $n^{\circ} 129-132$, janvier-juin 2014, p. 67-79 (version corrigée)}

Dans Le Signifiant imaginaire (1977), Christian Metz précise que l'expérience du film en salle s'apparente moins au rêve nocturne stricto sensu qu'à la rêverie diurne ${ }^{1}$. Il y a à cela plusieurs raisons majeures : le rêveur ne sait pas qu'il rêve, le spectateur sait qu'il est au cinéma (d'ailleurs, son corps n'est pas aussi oublié que celui du rêveur : il peut gesticuler, marquer son mécontentement, encourager bruyamment un personnage, etc. $)^{2}$; la perception filmique est « réelle » et n'est pas, comme le rêve, une perception relevant strictement du «psychisme interne $»^{3}$; enfin, le film narratif est considérablement plus logique que le moins illogique des rêves ${ }^{4}$. Ajoutons que le cinéma produit plus des «impressions » (au coefficient de leurre moindre) que des illusions, que le spectateur se heurte au fantasme d'autrui ${ }^{5}$ (l'instance énonciatrice du film) et que la projection en salle est publique et collective (là où le rêve est un état privé ${ }^{6}$ ). Avec Georges Perec, on pourrait affirmer que le rêve est ce film impossible - aberration de l'un à l'autre - dont à la fois je serais l'acteur principal, suivrais le tournage et assisterais à la projection ${ }^{7}$.

«L'état filmique » est, par rapport à «l'état onirique », un " mélange complexe d'affinités et d'écarts ${ }^{8}$. Si le film «favorise le retrait narcissique et la complaisance fantasmatique ${ }^{9}$, c'est sur un mode moins intense et plus en frontalité avec la réalité matérielle, et donc moins sûr et moins efficace que le

\footnotetext{
${ }^{1}$ La langue française signale explicitement la différence entre rêver de (les images produites par l'activité onirique inconsciente) et rêver à (songer, désirer, aspirer... : élargissement de la conscience éveillée).

${ }^{2}$ Christian Metz, Le Signifiant imaginaire. Psychanalyse et cinéma, Paris, Christian Bourgois, 1993, p. 123.

${ }^{3}$ Ibidem, p. 133.

${ }^{4}$ Ibid., p. 148. Le cas du cinéma expérimental serait différent. Mais on sait que la théorie de Metz porte sur le film narratif.

${ }^{5}$ Ibid., p. 123.

${ }^{6}$ Le rêve est, par cet aspect essentiel au moins, plus proche du Kinétoscope de Thomas Edison et W. K. L. Dickson (dès 1888), dans lequel un seul spectateur à la fois pouvait regarder les images à travers un œilleton, que du Cinématographe des frères Lumière...

${ }^{7}$ Georges Perec, La Boutique obscure, rêve n ${ }^{\circ}$ 14, Paris, Denoël, 1973, s. p.

${ }^{8}$ C. Metz, Le Signifiant imaginaire, op. cit., p. 130

${ }^{9}$ Ibidem, p. 130.
} 
rêve. Voir un film provoque plutôt une « baisse de la vigilance ${ }^{10}$. Baisse de la vigilance : Metz ajoute, en effet, que l'assoupissement menace plus pendant un film qu'avant ou après. Qui n'a jamais fait cette expérience, toujours un peu honteuse pour le cinéphile déclaré ? La situation psychique où le spectateur commence à s'endormir mais se rend compte encore qu'il voit ou lutte pour voir le film, rejoint alors - coincidentia oppositorum - celle où le rêveur se réveille et sait, ou sent, qu'il rêve ${ }^{11}$ in extremis dans le moment médian de la transition du sommeil au réveil, pourvu qu'un tel moment dure un peu (ainsi à l'ouverture de la Recherche proustienne) ${ }^{12}$.

De cette étrange expérience d'attention flottante, voire de franc assoupissement, Serge Daney a donné une explication on ne peut plus décomplexante, dans un article pour Libération (1989), et dans le contexte - il est vrai différent de la salle - de la retransmission d'un film à la télévision : mais son propos porte explicitement sur le rapport au film en tant que tel, et non sur son mode de réception (en partant du principe que les deux soient dissociables). Que dit Daney ? À l'exception des films irrémédiablement soporifiques et de ceux qui stimulent et tiennent éveillés par leur rythme, leur suspense, etc. ; à côté de cette partition qui catégorise les films selon le degré de tension qu'ils procurent, il existe un autre critère de démarcation suivant « un art méconnu de dormir à un film $»^{13}$. Face aux kilomètres de mauvais films, «inrêvables, ceux qui confondent l'art d'enchaîner les plans avec celui d'inventer de la durée ${ }^{14}$, on trouve parfois, dans les grands films, «qui ont su mettre le temps de leur côté », « un sommeil de bonne qualité » ${ }^{15}$. La sentence tombe : ne pas dormir devant, par exemple, Le Monde d'Apu (1959) de Satyajit Ray — on connaît l'admiration que Daney avait pour le cinéaste indien - est le signe d'un «mauvais coucheur qui ne sait rien du cinéma ${ }^{16}$.

\section{Bachelard}

${ }^{10}$ Ibid. - Raymond Bellour a comparé récemment l'expérience du spectateur en salle à celle de l'hypnose, un autre état intermédiaire entre la veille et le sommeil (Le Corps du cinéma. Hypnoses, émotions, animalités, Paris, P.O.L, 2009 ; par exemple pp. 59-63).

${ }_{11}$ C. Metz, Le Signifiant imaginaire, op. cit., p. 131.

12 Je laisserai délibérément de côté la question du rêve lucide, selon l'expression introduite dès 1867 par l'écrivain, sinologue et onirologue français, Léon d'Hervey de Saint-Denys (Les Rêves et les moyens de les diriger), puis repensée par la psychanalyse et notamment par le néerlandais Frederik Willems van Eeden (1913). Le rêve lucide, souvent décrit depuis Homère, Aristote ou saint Augustin, est un rêve dans lequel le rêveur a conscience qu'il rêve : ce qui revient à mettre en scène l'énoncé le rêve n'est qu'un rêve. Ce cas particulier ne me semble pas invalider la distinction structurante du présent texte — qui va désormais se déployer - entre rêve (endormi) et rêverie (éveillée) puisque, bien que la conscience de rêver soit supérieure dans le rêve lucide, que le rêve lucide revienne à formuler une critique du rêve comme celle que nous pourrions élaborer éveillés (Freud), j'y reste un rêveur endormi...

${ }^{13}$ Serge Daney, Devant la recrudescence des vols de sacs à main, Lyon, Aléas, 1991, p. 94.

${ }^{14}$ Ibidem, p. 95.

${ }^{15}$ Ibid., p. 94.

${ }^{16}$ Ibid., pp. 94-95. 
Que le spectateur s'endorme ou, sans aller jusque-là, somnole, rêvasse devant un film, quittant par la pensée la salle et le film matériel, n'est donc point honteux : après tout, la rêvasserie est encore l'une des formes de la rêverie éveillée dont Metz avait fait l'étalon de l'état du spectateur. La rêverie, plus que le rêve, doit d'ailleurs être mieux adaptée à la lumière tamisée de la salle de cinéma : à la différence des éclats de l'inconscient freudien, la rêverie — à suivre désormais Gaston Bachelard (qui y a consacré toute la dernière partie de son œuvre) - nous donne accès à un tout autre visage de notre inconscient, «l'inconscient tranquille, l'inconscient sans cauchemar, [...] le psychisme du clair-obscur $»^{17}$. Qui plus est, la rêverie, que nul ne cherche à expliciter, invite à rester plus fidèle aux images.

Le psychanalyste veut, en effet, faire toute la lumière sur les images. Mais l'image ne tient que de sa crevasse, de sa précarité, de son absentement : entre lumière et ombre, proximité et éloignement, apparition et effacement, etc. Pour la psychanalyse, l'image du rêve a une signification, elle s'inscrit dans une trame narrative, elle code un message de mots : il faut la casser pour décortiquer l'énigme verbale termite. "Il faut s'ingénier à trouver l'être vrai sous la caricature $»^{18}$. Les rêves, qui cryptent des pensées verbales en images, ne peuvent, à rebours, échapper aux mots et à la narration : ils ont besoin d'autres mots si nous voulons «les réhabit[er] pour nous convaincre qu'ils furent les nôtres ${ }^{19}$, et ces mots, d'ailleurs, les refont, les déforment, les défigurent — tant l'œuvre du rêve, ce sont images qui ébranlent le texte inconscient au point de le rendre illisible, et vont ainsi contre le texte...-, rajoutent des récits au leur, les manquent. Ce n'est jamais du rêve dont nous parlons, mais de notre récit sur ce récit, ou élaboration secondaire, dans laquelle nous mettons déjà, en récrivant les images, de la lisibilité, de l'intelligibilitée ${ }^{20}$. Le rêve, malgré les apparences, n'est peut-être qu'affaire de langage et de grammaire. Avec le rêve, a parte ante ou a parte post, on serait finalement cerné par le discours.

La rêverie, au contraire, tire jouissance de l'image pour elle-même : «Une rêverie, à la différence du rêve, ne se raconte pas $»^{21}$. Elle «travaille en étoile ${ }^{22}$. Elle ne cherche aucun rébus, aucun secret, aucune amande. Elle est une «poétique $»^{23}$. Rêverie et poétique mettent toutes deux l'accent sur la

\footnotetext{
${ }^{17}$ Gaston Bachelard, La Flamme d'une chandelle, Paris, PUF, coll. «Quadrige », 1961, p. 7. Je souligne.

${ }^{18}$ Ibidem, p. 11.

${ }^{19}$ Idem, La Poétique de la rêverie, Paris, PUF, coll. «Quadrige », 1960, p. 10.

${ }^{20}$ Le cinéma, en filmant des rêves qui se font, prétend revenir en deçà de cette narration seconde. Faut-il ajouter qu'il n'y parvient évidemment pas ?

${ }^{21}$ G. Bachelard, La Poétique de la rêverie, op. cit., p. 7. Je m'écarte, ici, des propositions metziennes pour qui la rêverie - dans la lignée de Freud - est un fantasme onirique, c'est-àdire une histoire. Ce qui explique, pour Metz, le succès du cinéma narratif. C'est encore juger de la rêverie à partir $d u$ rêve (qui raconte une histoire). La suite immédiate de cet article posera, a contrario, que la rêverie n'est pas une « dérivation du rêve » (ibidem, p. 10).

${ }^{22}$ G. Bachelard, La Psychanalyse du feu, Paris, Gallimard, coll. « NRF Idées », 1949, p. 32.

${ }^{23}$ Idem., La Flamme d'une chandelle, op. cit., p. 11.
} 
«vertu d'origine» des images ${ }^{24}$. L'image — dans un geste esthétique qui voudrait remonter, autant qu'il est possible, à notre agrément originaire, préobjectif et antéprédicatif avec le monde, avec le premier déferlement prérationnel du sensible - est ce par quoi nous pouvons encore essayer d'entrer en « contact muet avec les choses, quand elles ne sont pas encore des choses dites $»^{25}$.

La rêverie n'est pas un rêve affaibli, sans récit, sans mystère. En sous-régime. L'originalité de la rêverie tient à ce que la conscience y veille encore. C'est la conscience qui s'y poétise. "Le rêveur de la rêverie sait que c'est lui qui s'absente $»^{26}$. Le rêveur profondément endormi, pour sa part, ne peut pas se représenter ni dire qu'il est en train de rêver : à plus d'un titre, Je ne rêve pas. «Le rêve de la nuit est un rêve sans rêveur ${ }^{27}$. Ce n'est pas hasard si Freud parle de «l'ombilic du rêve» pour désigner le point «impossible à reconnaître ${ }^{28}$ en lequel s'arrête le rêve, où rien de plus ne peut être ajouté, « une pelote de pensées oniriques qui refusent de se laisser démêler, [...] le point par lequel [le rêve] est assis sur l'inconnu » ${ }^{29}$, d'où le rêve surgit " comme le champignon de son mycélium $»^{30}$ : comme si l'origine du rêve se situait, métaphoriquement, dans la naissance, c'est-à-dire précisément avant l'apparition de toute forme de conscience de soi.

Les réflexions du dernier Bachelard sur la rêverie portent presque exclusivement sur l'image littéraire, et tout spécifiquement dans l'écriture des poètes : c'était sa passion de lecteur, et sa seule compétence déclarée dans le cadre d'une phénoménologie de l'art. Ce n'est pas en tant que la poésie dit qu'elle intéresse le philosophe, c'est en tant qu'elle donne à voir: vibrations, ébranlements, détraquement, tétanie... d'ailleurs, Bachelard ne cite jamais un poème en entier (ce serait rester trop «branché » sur la narration) : mais des strophes, des vers, des hémistiches - des coupures (dans le texte, du texte). Il ne serait donc pas tout à fait illégitime - malgré des aspects de la méthode moins électrisants - d'en étendre, comme je viens de le suggérer, quelques procédures et résultats à l'image visuelle, et en l'occurrence au film.

Travaillant sur tel corpus pour d'autres réflexions, mon choix s'est porté sur quelques films de Jean Renoir, qui m’ont paru agréer des exigences très originales de rêverie comme donnant forme (partielle) au film — non pas par la représentation imagée de rêverie à l'écran mais par une sorte de rêverie imageante produisant des événements d'images intensifs.

\footnotetext{
${ }^{24}$ Id., La Poétique de la rêverie, op. cit., p. 2.

${ }^{25}$ Maurice Merleau-Ponty, Le Visible et l'Invisible, Paris, Gallimard, coll. « Tel », 1979, p. 61. Il y a de la phénoménologie dans la méthode de Bachelard. Il s'en explique longuement.

${ }^{26}$ Ibidem, p. 129.

${ }^{27}$ Ibid., p. 20. Hormis quelque fois, in extremis, dans le demi-sommeil de la fin de nuit: mais encore n'est-ce pas en tant que rêvant que je me sais rêvant, c'est en tant que m'éveillant.

${ }^{28}$ Sigmund Freud, L'Interprétation des rêves, trad. I. Meyerson, éd. revue et augmentée par Denise Berger, Paris, PUF, 1976, p. 103.

${ }^{29}$ Ibidem, p. 446.

${ }^{30}$ Ibid.
} 


\section{Jean Renoir 1}

Première remarque. Plusieurs films renoiriens portraient des individus rétractés dans leur rêverie éveillée - le poète excentrique de Tire au flanc (1929), le clochard bougon de Boudu sauvé des eaux (1932), l'épouse insatisfaite de Madame Bovary (1933), l'adolescente amoureuse du Fleuve (1951), etc. Un seul exemple, représentatif de son art à maturité, me suffira pour montrer l'importance décisive de la question de la rêverie quand elle provoque, au-delà de ce qui est représenté à l'écran, une forme filmante.

À l'ouverture du Crime de Monsieur Lange (1936) quand, dans le récit-cadre, Valentine demande à Amédée s'il dort, celui-ci répond qu'il se repose. Autrement dit : il est bel et bien conscient (il parle), mais d'emblée, le film à peine commencé, son personnage principal se déconnecte, s'absente, courtcircuite ses attaches au monde (d'autant qu'il voudrait probablement le réviser : il vient de tuer un homme...), «premier habitant du monde de la solitude ${ }^{31}$. Tout au long du film (le flash-back), le personnage de Lange, salarié d'une petite maison d'édition parisienne, sera défini par la rêverie, la rêvasserie (simple différence de degré de la rêverie la plus raffinée à la rêvasserie la plus informe ; aucune de nature). Lors du premier dialogue avec Valentine, dans l'ordre chronologique de l'intrigue, il avoue: "Quand j'écris, c'est comme si je dormais ». Écrire, c'est rêver (le rêve est la mise en fiction du sommeil) ; mais, tout aussi bien, rêver sans dormir.

À tel point que Valentine s'y méprend, et le prend par le mauvais bout, en le traitant de «somnambule». Plus tard, son escroc de patron - Batala - le qualifiera de «rêveur» et dira à Valentine, une ex-conquête, qu'elle a besoin d'un «rêveur» comme amant : elle s'entichera précisément d'Amédée Lange. Et ainsi de suite. Les exemples, dans les dialogues (coécrits avec Jacques Prévert), ne manquent pas. Comment également, surtout — retour à l'image - ne pas voir tous ces plans où, discutant avec l'un ou l'autre, Lange se distrait, parfois d'un simple coup de tête furtif, donne l'air de ne plus écouter, répond à peine, détourne négligemment le regard, se perd dans la douceur benthique de sa rêverie ? C'est que — Valentine a tôt averti qu'il " était toujours ailleurs » Lange est un as de l'évasion par l'imagination. Son esprit va aux cow-boys et aux peaux-rouges, à ces histoires du Far West (où il n'a jamais mis les pieds); à cet «Arizona Jim»dont il écrit inlassablement les aventures abracadabrantes sur le modèle des romans à trois francs six sous de la littérature populaire américaine (dime novels), mais aussi - même s'il n'y est jamais fait allusion explicitement : médium oblige - des débuts du western au cinéma (avec Tom Mix, William S. Hart). Amédée Lange ne veut pas quitter les rêveries solitaires de l'enfance - celles qui «nous ont donné les immensités primitives », où «l'image prime tout ${ }^{32}$. C'est que l'enfant n'a pas besoin de grandes choses, contrairement à l'adulte, pour voir grand. En en prolongeant les rêveries, Lange,

${ }^{31}$ G. Bachelard, La Poétique de la rêverie, op. cit., p. 87.
${ }^{32}$ Ibidem. 
modeste employé timide, continue à vivre «dans un dedans qui n'a pas de dehors $»^{33}$. «Le monde ne lui fait plus de vis-à-vis ${ }^{34}$ : par la rêverie, Lange qui confesse ne jamais sortir, ou rarement - accueille en lui le monde, et le plus exotique, le plus lointain (far), bouclant le moi et le monde en une sorte de bande de Möbius mentale.

Le Crime de Monsieur Lange est film de l'adieu à l'enfance et de l'entrée dans l'âge adulte : par le désir sexuel (Edith, Estelle, Valentine), puis par l'assassinat (Batala). L'enfant, déjà, barbote avec des pulsions destructrices et sexuelles ; il cherche à les satisfaire - l'enfant est essentiellement plaisir - mais n'est pas physiquement capable de passer à l'acte. L'adulte, tout en ayant appris à mieux les assouplir (maîtriser, canaliser), pourra aussi mieux les assouvir. Et l'âge adulte, plus inscrit dans le réel, abîmera la rêverie.... Il faut bien voir, dans le meurtre de Batala, une parodie de duel de western, inégal et lâche, où le " héros » - bien terne - ne laisse aucune chance à son adversaire (la futur victime n'est pas armée) et manque de noblesse et de grandeur d'âme (même si l'autre est une crapule : la vraie magnificence, justement, s'y mesure). Amédée Lange n'est pas le valeureux et loyal «Arizona Jim » auquel il prêtait sûrement ses traits, dans l'idéal de la rêverie : cette fiction ne pouvait tenir que par des survivances d'enfance. Ce moment, si important pour Lange, où il aurait pu superposer la rêverie et la réalité (la rêverie, je l'ai rappelé, indistingue le dedans et le dehors...), mais s'il se fût tout autrement comporté (le pouvait-il seulement : l'acte anéantit la rêverie), n'est pas seulement une déception : c'est l'expulsion atroce de la rêverie hors d'un corps.

Et, donc : le célèbre panoramique circulaire de la scène du meurtre, sur lequel on a tant écrit, et dont il paraît impossible d'extraire quelque nouveauté ${ }^{35}$. Et pourtant. N'est-il pas significatif que, au moment où Amédée s'apprête à quitter définitivement l'enfance et les époques de la rêverie, où il va à l'encontre de la rêverie en marchant vers l'acte qui le jettera dans l'âge adulte, cette rêverie coulisse de son corps au film, le seul - peut-être - encore capable de la recevoir, s'il est vrai que «le cinéma est né (comme un enfant) d'une espèce de répétition publique du rêve intime ${ }^{36}$ ? N'est-il pas envisageable de voir dans le mouvement de la caméra qui, investissant visuellement le motif de la boucle, se détourne de la ligne droite attendue (travelling de suivi, par exemple) et quitte son personnage (qu'elle rejette ipso facto hors du champ), le même geste de distraction que Lange avait, de temps à autre, avec ses interlocuteurs? La rêverie n'y devient-elle pas rêverie $d u$ film ?

Que faire de telle hypothèse ?

\section{Kuntzel}

\footnotetext{
${ }^{33}$ Ibid., p. 144.

${ }^{34}$ Ibid.

${ }^{35}$ Voir notamment André Bazin, Jean Renoir, Paris, Champ Libre , 1971, pp. 40-42.

${ }^{36}$ Jean Louis Schefer, Du monde et du mouvement des images, Paris, Cahiers du cinéma, coll.

«Essais », 1997, p. 5.
} 
Je rembobine un peu pour revenir, quelques instants, sur le rêve nocturne. Que le film travaille de lui-même - sans la moindre intervention de l'instance créatrice consciente (cinéaste, chef opérateur, monteur, etc.), transcendante et étrangère - sur le même modèle que le rêve, est un fait théorique établi depuis deux articles déterminants $(1972,1975)$ de Thierry Kuntzel sur le «travail du film» (représentatif-narratif : comme l'est le rêve); auxquels il convient d'adjoindre un troisième, sur le défilement filmique (1973).

Pour Kuntzel, le film, bien qu'il n'ait pas de conscience, n'en a pas moins un inconscient. Pareille affirmation, sur le principe, n'a rien de bien paradoxal si l'on se rappelle que l'inconscient freudien n'est pas défini relativement à la conscience, n'est pas la conscience en négatif et qu'il possède ses lois et sa logique propres. Kuntzel ne fait que porter à l'exposant cette affirmation : si l'inconscient n'est pas l'autre de la conscience, alors il n'est pas besoin de la moindre conscience pour qu'il y ait inconscient. Au demeurant, toute notre vie ne commence-t-elle pas par être uniquement inconsciente (seconde topique) ? La conscience n'apparaît-elle pas comme différenciation supplémentaire à partir de l'inconscient?

Parler de «travail du film», c'est poser entre rêve et film certaines similitudes: non dans les contenus figurés, mais dans le modus operandi figurant. Si le film, comme le rêve, d'un côté fait subir à des pensées verbales des métamorphoses imageantes ${ }^{37}$, la figuration, pour Kuntzel, n'est pas que la traduction d'un texte préexistant. Quoiqu'il s'inscrive dans la méthodologie textuelle de la sémiologie, par opposition au « fanatisme du 'visuel' qui confine au déraisonnable ${ }^{38}$, Kuntzel — c'est l'un de ses apports principaux - va y intégrer une attention aux processus imageants de figuration et y greffer une démarche plus esthétique (significatives sont les références à Lyotard apparaissant avec «Le travail du film 2 »), l'éloignant des thèses de la réduction de l'image à la structure d'un langage. Le travail du rêve n'est pas un langage mais la violence que la figure exerce contre le langage. "Prélever, mettre en contact, franchir la barre qui sépare les 'disciplines', troubler, pervertir, mixer peut-être enfin trouver une méthode suffisamment labile pour dire du film la fluidité, le mouvement, la mixité, la spécificité du processus signifiant ${ }^{39}$.

Le «travail du film» exige la distinction entre un contenu manifeste et un contenu latent du film. À l'instar du rêve, le film procède figuralement par travestissements et déguisements (déplacement, condensation). L'analogisme photographique du cinéma n'est qu'un leurre et empêche de voir ce qui, dans l'image, fait sens: «une trame textuelle, une pratique sémiotique spécifique, une figuration ${ }^{40}$. Qui dit «latent , dit par ailleurs «inconscient ${ }^{41}$. Et

\footnotetext{
${ }^{37}$ C'est la fameuse «Rücksicht auf Darstellbarkeit » - « prise en compte de la figurabilité », " souci de la représentation figurative », « égard aux moyens de la mise en scène » (Jacques Lacan) : l'expression est difficilement traduisible.

${ }^{38}$ C. Metz, Langage et cinéma, Paris, Larousse, 1971, p. 24.

${ }^{39}$ Thierry Kuntzel, « Le travail du film 2 », Communications, 23, 1975, p. 183.

${ }^{40}$ T. Kuntzel, « Le travail du film », Communications, ${ }^{\circ} 19,1972$, p. 39.
} 
l'inconscient du film, c'est le photogramme. Lorsque le film est projeté, le mouvement apparaît, et le photogramme qui est pourtant le point de départ matériel pelliculaire de la fabrication du film est refoulé : à la fois nié (par l'image animée) et repoussé en arrière (par le défilement). Quand la pellicule passe, les photogrammes défilent, et « se défilent »: on ne voit pas, sur l'écran, des images fixes défiler, mais une même image varie, par superposition; les claps de l'occultation de l'objectif du projecteur interdisant, par ailleurs, toute persistance rétinienne (sinon l'image ne serait qu'un «embrouillamini d'images rémanentes ${ }^{42}$ ). Entre le «film-projection » et le «film-pellicule» (distinction venue de Bergson $)^{43}$, le photogramme appartient à cet « espace à la fois matériel et mental, fondé sur la trace mnésique, sa disparition et sa rémanence, son effacement et sa réinscription ${ }^{44}$. Mais, n'en continuant pas moins d'agir en silence, ou à l'aveugle, les photogrammes repoussés — constituant l'inconscient du film - vont être à l'origine de phénomènes d'échos, de rimes, de vibrations, de cloques (de certains plans, scènes, épisodes à d'autres), lesquels agissent par places comme un véritable retour du refoulé. Le spectateur accort les apercevra ; ou pas. De tels phénomènes de gondolement, de résurgence, de gaufrage sensibles impliquent, en effet, que des traits, des motifs, et ainsi de suite, soient retenus, i. e. captés et immobilisés, malgré le défilement, d'une image à une autre, parfois très éloignée de la première, dans le temps déroulant du récit.

Un film se présente donc, tel le rêve, comme un ensemble d'éléments constituant une chaîne de significations à partir d'images qui poussent depuis

${ }^{41}$ Je rappelle la différence exacte entre latent et refoulé. « Nous savons cependant qu'il existe deux variétés d'inconscient : les faits psychiques latents, mais susceptibles de devenir conscients, et les faits psychiques refoulés qui, comme tels et livrés à eux-mêmes, sont incapables d'arriver à la conscience » (S. Freud, Essais de psychanalyse, trad. S. Jankélévitch, Paris, Payot, coll. «Petite Bibliothèque », 1989, p. 179). Le refoulé, seul, est à l'origine de la formation du rêve, sans pouvoir jamais passer dans la conscience : la résistance, même la conscience assoupie, est bien trop forte. Il ne le pourra qu'indirectement, par l'intermédiaire de pensées latentes à lui attachées et qui, pour inconscientes qu'elles soient (au sens défini cidessus), relèvent d'un état psychique médian, lequel pourra plus aisément permettre l'accès à la conscience, sous la condition néanmoins de certaines procédures métamorphiques.

42 Jacques Aumont, L'Image, Paris, Nathan, coll. « Nathan Cinéma », 1990, p. 34.

${ }^{43}$ Voir T. Kuntzel, «Le défilement », Revue d'esthétique, n 2-3-4, 1973, pp. 97-110. Une précision. Le photogramme du film, pour statique qu'il soit, n'est pas comme la photographie d'une pose tenue dans le temps, donc immobile. Le déplacement de tel objet ou tel corps devant la caméra fait que «le photogramme cinématographique, extrait d'une réalité mouvante, comporte inscrit en lui, à la différence de la photographie, l'amorce du mouvement, des signes flous qui, se superposant plutôt que se succédant, convergent vers l'impression d'un dynamisme avant de servir une succession»: Dominique Chateau, Philosophie d'un art moderne : le cinéma, Paris, L'Harmattan, coll. "Champs visuels », 2009, p. 65. Si les photogrammes se succèdent «dans le film inerte », les images projetées «ne se succèdent pas horizontalement, mais, par rapport au plan de l'écran, se superposent verticalement pour donner une impression de continuité » (p. 66. L'auteur souligne).

${ }^{44}$ R. Bellour, L'Entre-images. Photo. Cinéma. Vidéo, éd. revue et corrigée, Paris, La Différence, coll. «Les Essais », 2002, p. 308. 
son for intérieur cryptique, qui « figurent un terme absent ${ }^{45}$. Non seulement il existe une perception figurative des images par des procédures ni conscientes ni conceptuelles (l'idée n'est pas neuve), mais les images «pensent entre elles ${ }^{46}$.

Écrire - le texte sur le film - consiste alors à faire à peu près ce que les mots matinaux font avec les rêves de la nuit qui, comme le film, «se dévident en dehors de moi, sans intervention possible de ma part ${ }^{47}$. Le texte permet de mieux voir le film. Kuntzel nomme cette opération «visionner un film» (par opposition à le voir, ou le revoir : aucune différence significative entre les deux) : soit « ralentir ou arrêter le mouvement (la continuité) pour repérer l'immobilité (la discontinuité) qui le soutient, isoler des motifs visuels ou sonores, les confronter par un retour en arrière [...], écouter-voir le film comme aucun usager du cinéma ne peut le faire, récrire le spectacle sous forme de texte décaper les couches d'opacité référentielle qui occultent le travail de signification $»^{48}$. Ailleurs : «Le spectacle, dans sa successivité, ne permet pas l'accès au géno-texte ; celui-ci ne peut être approché que lorsque le spectacle est transformé par un travail de visionnement - arrêt, cassure, revision -, travail qui, seul, permet d'amorcer une lecture $»^{49}$.

La renonciation aux prérogatives de la conscience, ou des consciences créatrice(s) dans le «travail du film» de fiction est plus conforme au principe même du travail intégralement inconscient du rêve : lesdites consciences étant toujours réveillées quand on tourne... Contradiction particulièrement cinglante dans les scènes où l'on veut obtenir un «effet rêve », et où les consciences interventionnistes veulent marquer leur différence, c'est à dire leur singularité par trop d'esthétisme et d'abstraction ${ }^{50}$, là où le rêve reste fondamentalement narratif et représentatif (c'est la condition de la satisfaction imaginaire), c'est-àdire plutôt exactement du même registre que le reste du film... Nul rêve fait par un homme ne ressemble aux rêves les plus visuellement débridés que nous a donnés le cinéma : flashes stroboscopiques, couleurs clignotantes, décors peints, carton-pâte, surimpressions, animation image par image, dessin animé, etc. Le rêve devient alors un moyen - usé jusqu'à la corde - de «faire art », tel moment, dans un film obéissant en son ensemble au régime de l'analogie photographique référentielle. Mais le procédé est vicié : sous couvert de mettre en avant le spectacle sensible et iconique de l'image, la représentation du rêve dans le cinéma narratif est en réalité serve de la plus coercitive des narrativités, puisque le scénario, donc du verbal, du récit — et, au-delà, les

\footnotetext{
${ }^{45}$ T. Kuntzel, « Le travail du film », op. cit., p. 33.

${ }^{46}$ Hubert Damisch, «La Peinture prise au mot», Critique, n 370,1978 , p. 289. Je souligne. Je reporte à une autre occasion la discussion sur l'adéquation du lexique de la pensée ici.

${ }^{47}$ T. Kuntzel, « Le travail du film », op. cit., p. 27.

${ }^{48}$ Ibidem.

${ }^{49}$ Idem, « Le travail du film $2 »$, op. cit., p. 148.

${ }^{50}$ Il y a des exceptions : notamment quand le rêve - alors semblable, dans son rendu visuel, aux autres scènes de la diégèse, et en l'occurrence, dans le film représentatif et narratif, à n'importe quel épisode de la vie réelle - doit passer, pendant un laps de temps plus ou moins long, pour une péripétie effective de la fable.
} 
codes des genres, l'accord des producteurs, etc. - prévoit la scène en question et la justifie au nom de la sacro-sainte vraisemblance psychologique. Le déchaînement y va, finalement, dans le sens de l'enchaînement. Or le rêve, comme l'écrit Jean-François Lyotard, «n'est pas la parole du désir, mais son œuvre ${ }^{51}$; il consiste en la «transgression ${ }^{52}$ de l'ordre de la parole. De ces quelques remarques amorcées, on peut dégager deux questions :

1) N'est-il pas envisageable de poser, outre le rêve, qu'il puisse exister un travail de la rêverie par le film, à savoir que le film deviendrait comme son propre spectateur, un spectateur distrait, qui somnole, se laisse aller devant ses images ?

2) Cette rêverie immanente des images n'est-elle pas ce qui, dans certaines scènes de rêve (l'objet d'étude du présent volume), va marquer justement le détournement du film du moment pénible (parce que contre-onirique) de la représentation le plus souvent largement mimétique du rêve par le cinéaste et ses adjoints (d'où sa distraction...), permettant ainsi, par tel déplacement, de rester fidèle à la méthode (figurale) du rêve?

\section{Jean Renoir 2}

Les scènes de rêve stricto sensu ne sont pas nombreuses dans la filmographie de Renoir. Deux durant la période muette «expérimentale» — dans La Fille de l'eau (1925) et La Petite Marchande d'allumettes (1928) - quand le cinéaste débutant bricole des films au fort amateurisme et s'enthousiasme pour les trucages du cinéma ${ }^{53}$. Elles cèdent la place, par la suite, à quelques séquences de tournure " oniriste », plutôt que des rêves à proprement parler. «Ce n'est pas moi qui ai exclu les rêves de mes films postérieurs à La Petite Marchande d'allumettes, ce sont les producteurs. Le rêve les effraie. J'ai eu plusieurs projets de films contenant des rêves, mais aucun n'a pu être tourné $»^{54}$.

Le rêve de Gudule (ou Virginie suivant les copies) dans La Fille de l'eau, second ou premier opus du cinéaste - selon le sort que l'on réserve à Catherine (1924) - , alors que la protagoniste est endormie sous un arbre pendant que fulmine un violent orage, est encore complètement abandonné à la conscience vigoureuse des effets visuels : pouvait-il en aller autrement pour un cinéaste qui, à concurrence de l'avoir fait pour la carrière d'actrice de son épouse (Catherine Hessling), a déclaré s'être lancé dans le cinéma par entichement pour les trucages ? Le film ne lésine pas en chemin : ralentis, montage inversé, images culbutées, anamorphoses, objets insolites et jeux perspectifs (caméléon) - et ainsi de suite.

\footnotetext{
${ }^{51}$ Jean-François Lyotard, Discours, Figure, Paris, Klincksieck, coll. « Esthétique », 1971, p. 239.

52 Ibidem.

${ }^{53}$ On sait l'impact qu'ont eu sur lui les trouvailles visuelles du Brasier ardent (1923) de Ivan Mosjoukine, dont l'intrigue repose sur les rêves d'une jeune femme et qui brouille les différents registres ontologiques de la réalité et du rêve.

${ }^{54}$ Entretien avec Jean Renoir, URJC-Informations, Toulouse, 1962. Cité in Pierre Haffner, Jean Renoir, Paris, Rivages, coll. « Cinéma », 1987, p. 59.
} 
S'agissant de la couleur blanche, très présente, elle y reste au service des pouvoirs narratifs - innocence, chasteté, pureté : Gudule, ou « les infortunes de la vertu » (c'est ce que dit très exactement le scénario), rêvant à un « chevalier blanc » de conte de fées - et ne fait jamais la moindre embardée, d'emblée affichée par les dehors de l'image : surexposition, vêtements des protagonistes, etc. Intéressant, à ce titre, est le moment de chevauchée, emportant Gudule et Georges, que le cinéaste introduit dans le rêve (Fig. 1). Le tournage de cette séquence a nécessité un cylindre géant sur lequel a été montée une plateforme pivotante permettant d'enregistrer le déplacement en cercle du cheval ; puis les plans ainsi obtenus ont été incrustés en double impression sur un fond mobile (arbres, nuages). L'effet n'est pas anodin et entend constituer une sorte de traduction de l'étrangeté figurale du rêve: l'arrière-plan est net, mais l'avantplan (les figures) transparent, là où la technique usuelle de la rétroprojection produit des valeurs de netteté antipodales et plus conformes au réalisme de la perception perspective (structurée par le discours). 
Fig. 1

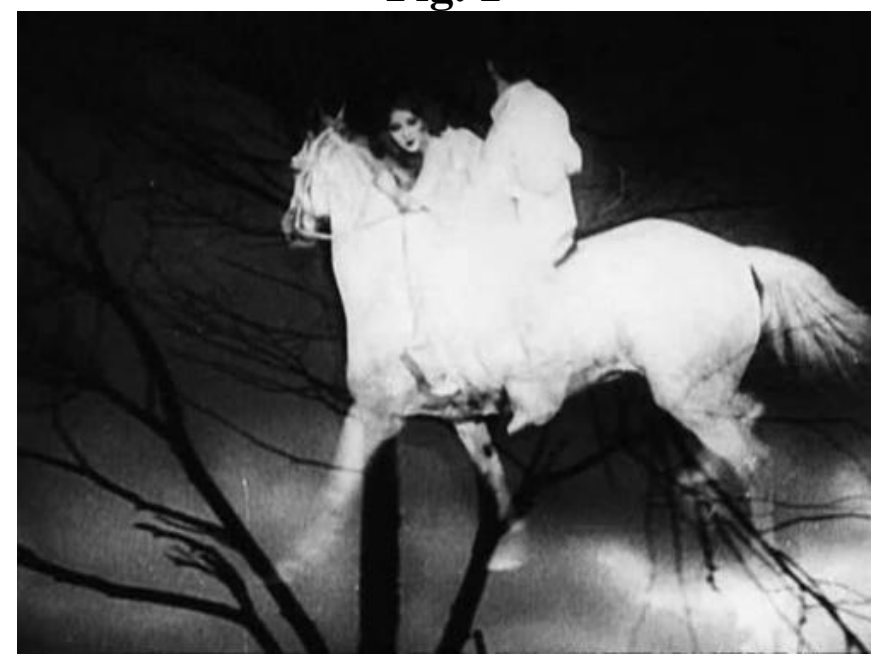

Jean Renoir, La Fille de l'eau, 1925

Très différent est le rêve de La Petite Marchande d'allumettes (d'après Andersen), cosignée avec Jean Tedesco, aux effets visuels nettement plus sobres (surtout d'éclairage et de pellicule ${ }^{55}$ ), qui est la justification principale du film. Ce rêve, à nouveau de sommeil, de belle étoile et de mauvais temps (pas question de lit chez Renoir : l'intempérance onirique est une intempérie sous un crâne), est d'abord plus complexe. Un épisode initial, où domine l'insouciance, montre Karen miniaturisée dans un magasin de jouets où les marionnettes, les peluches et les soldats de bois, se mettent à prendre vie. Elle y rencontre un jeune soldat (au visage d'un passant croisé un peu auparavant). Dans la seconde partie, funeste, l'officier veut protéger Karen de la Mort (qui a pris l'apparence d'un policier, travesti en hussard noir à ressort), et la transporte dans les nuages. Commence une furieuse chevauchée - quatre ans après La Fille de l'eau - au terme de laquelle le soldat est tué, et Karen emportée par son poursuivant fatal. Celui-ci la dépose au pied d'une croix et se débarrasse d'un de ses cheveux resté accroché à son dolman. Le cheveu vient se poser sur la croix qui se transforme en rosier; les pétales des roses (des flocons de neige dans le monde réel) tombent sur le sol... où repose le cadavre gelé de la jeune femme. (Tout au long du rêve, on aura noté des réminiscences climatiques (fantastiques) typiquement germaniques - un peu de cinéma expressionniste, peut-être ; mais plutôt, en deçà, le romantisme allemand: Hoffmann (L'Homme au sable), Arnim (Marie de Melïk-Blainville), surtout, plus exactement pré-romantique, La Balade de Lénore de Bürger, notamment par le truchement du tableau Les Morts vont vite, d'Ary Scheffer, dont les valeurs chromatiques sont ici inversées (Fig. 2).)

${ }^{55}$ Une pellicule panchromatique pour des intérieurs. 
Fig. 2

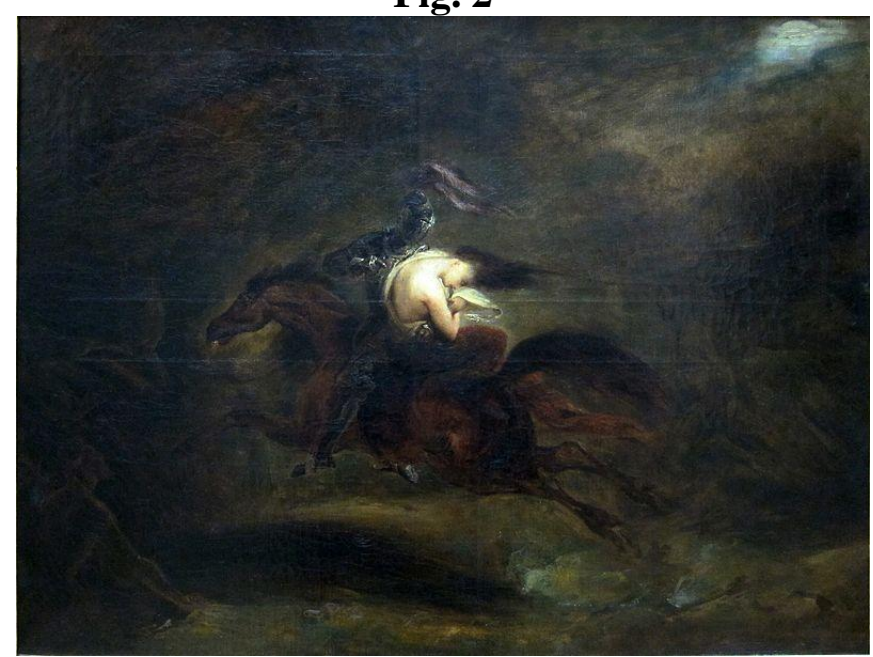

Ary Scheffer, Les Morts vont vite, c. 1830

On peut aisément voir dans chacun de ces deux épisodes une mise en abyme de phénomènes typiquement cinématographiques: d'une part, le gigantisme de la projection des images et les corps des spectateurs réduits à «des objets plus petits que les objets ${ }^{56}$; d'autre part, la projection de figures (sombres) en mouvement - galop infernal, décradages brutaux... - sur l'écran blanc. Que le fait ait été présent à la conscience de Renoir (voire de Tedesco) n'a, en l'occurrence, absolument aucune espèce d'importance. Le film se met en scène : il n'en était rien encore dans La Fille de l'eau. Il ne s'agit pas de voir la caméra (ici) comme le prolongement (l'outil) d'une conscience humaine - son relais, son assesseur - mais comme ce qui fait voir «les choses à partir de l'espace du film $»^{57}$, donc depuis le parage d'une absence de conscience : toujours du côté de l'image. Lors de la cavalcade, la caméra cherche à plusieurs reprises à photographier le plus de blanc possible — celui du fond monochrome de l'image: coton des nuages; par des procédures diverses: contre-plongée, cadrage biscornu, avance ou retard sur le raccord (avant ou après que les personnages entrent dans le champ). Ou encore : voilà comment le film, à ce moment-là, affecte. Cette chevauchée est en tout point chromatiquement contradictoire avec celle proposée par La Fille de l'eau, où les personnages étaient vêtus de blanc et les nuages, noirs (l'orage). (Fig. 3-4)

\footnotetext{
${ }^{56}$ J. L. Schefer, L'Homme ordinaire du cinéma, Paris, Cahiers du cinéma / Gallimard, 1980, p. 29.

${ }^{57}$ Béla Balázs, L'Esprit du cinéma, Paris, Payot, coll. « Petite Bibliothèque », 2001, p. 184.
} 
Fig. 3-4



Jean Renoir, La Petite Marchande d'allumettes, 1928

La couleur blanche était déjà très présente dans le magasin de jouets : gaze du voile à l'arrière-plan, robes des danseuses mécaniques, poupées de porcelaine, moutons en cartons (Fig 5)... Le motif visuel du blanc, qui s'impose dans le champ (parfois dans sa totalité) malgré le récit - la lisibilité des plans et de leur succession est bouleversée - tire l'image vers l'arrière et tisse, entre les plans, des liaisons vectorielles et figurales. La distraction n'est plus celle d'un regard qui s'égare (Le Crime de monsieur Lange) mais d'un regard - caméra plus fixe oblige également - comme hagard, éberlué (du point de vue de la logique narrative, qu'on quitte précisément...), ne suivant plus le cours des événements étrangers (scénaristiques), et dont l'attention au blanc marque avant tout qu'il est obnubilé par sa propre intériorité.

\section{Fig. 5}



J. Renoir, La Petite Marchande d'allumettes, 1928

En refusant d'enchaîner sur le rêve, de s'enchaîner à son cul-de-sac représentationnel (l'inconscient d'un personnage n'a que la consistance visuelle que les consciences fabricatrices lui prêtent), le film va réintroduire, par la 
rêverie, de la (dys)figuration onirique dans un registre d'images dont la production par des hommes est tout sauf « rêveuse » (les trucages pléthoriques exigent, bien au contraire, un niveau élevé de vigilance de la part des consciences impliquées), et dont le résultat visuel n'est jamais, du point du vue du travail du rêve, qu'une "escroquerie ». Contre les intentions manifestes et conscientes du cinéaste, ou pour le moins sans y prêter la moindre importance, la rêverie de La Petite Marchande d'allumettes travaille dans le sens d'un complet renversement des priorités figuratives : le blanc d'arrière-plan, qui, dans le rêve de Karen (l'image voulue par Renoir), est au bout de l'image (les trois protagonistes sont habillés de noir), devient, dans le travail de rêverie du film, la figure (Fig. 6) ; figure, pure intensité chromatique, image vierge non écrite, qui va, mieux que nulle autre marquer que le rêve, a justement à voir avec le «blanc » qu'est l'inconscient ${ }^{58}$. Alors que le rêve élaboré par le cinéaste tend du côté des effets saillants (surimpression, stop motion, etc.), le film, quant à lui, va comme épurer cette représentation fallacieuse, et raccorder sur la logique onirique de déformation du verbal, du scénario, en connectant sans la moindre délibération, par en-dessous la pensée de la mort, c'est-à-dire la téléologie intentionnelle du récit, la présentation de la vie (au moins dans la symbolique occidentale majoritaire du blanc — voir également la floraison, et déjà les jouets de la première partie...) plus adéquate à la situation de la petite vendeuse que les figures noires, hussard, chevaux, croix de la fable : puisque pour elle, rêvant (le geste filmique nous invite à nous identifier à elle : nous « sommes » «dans » sa tête), elle ne meurt pas - d'autant que le rêve (image sans cadre d'ailleurs) n'a pas vraiment d'interruption : le fondu enchaîné des pétales sur les flocons le fait plutôt déborder de ses contours comme le coloriage d'un dessin d'enfant.

Fig. 6

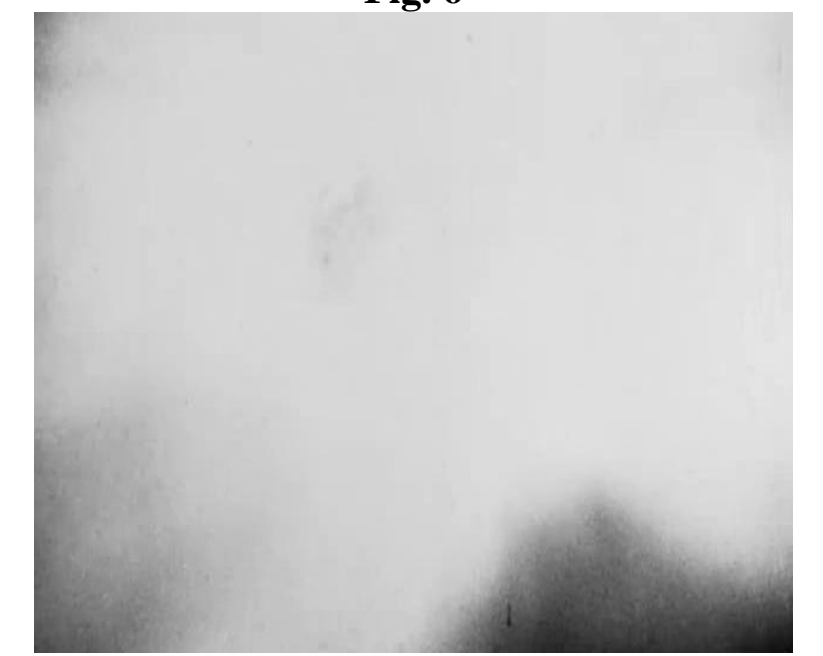

J. Renoir, La Petite Marchande d'allumettes, 1928

Le rêve de La Petite Marchande d'allumettes peut être vu comme le négatif jusques à l'acception photographique du terme - de celui de La Fille de l'eau :

58 «L'inconscient est le chapitre de mon histoire qui est marqué par un blanc... » (J. Lacan, Écrits, Paris, Seuil, coll. « Le Champ freudien », 1966, p. 259). 
beaucoup plus pauvre du point de vue de la représentation, et quantitativement parlant, cherchant moins à contrefaire le rêve que son prédécesseur ; mais c'est précisément cette pauvreté qui explique la qualité de la disponibilité spécifique de ses images à la rêverie du film - le cinéma n'aura, de toute façon, jamais été dans cette manigance de la ressemblance... Cette rêverie provoque, en totale indépendance avec les réquisits du récit et de la fable, la défiguration de la mimèsis plagiaire du rêve (de ses résultats en impasse : les images, un certain type d'images) et, partant, reconquiert l'orientation médullaire de l'activité onirique. Et du cinéma : la rêverie du film, qui se détourne du rêve représenté, cesse de traiter les images du rêve - y compris les transparences, les surimpressions... - comme des solides (en contradiction également avec les « substances évanouissantes ${ }^{59}$ du cinéma).

Renoir sera finalement obligé, par des producteurs peu convaincus, d'abandonner les images de rêves? Il n'y a sans doute pas à le regretter. Comme l'a montré Le Crime de monsieur Lange - mais on en dirait tout autant d'autres films -, la rêverie pourra ainsi mieux se détacher du rêve ponctuellement figuré (désormais nul) pour devenir pleinement imagination figurante diffuse. Jean Renoir n'est pas encore lui-même dans sa période muette : pour cela, il devra prendre la voie de l'intrigue et du jeu des acteurs, en tournant le dos à « l'avantgardisme » visuel de ses débuts (qui était de toute façon un trait de l'époque) et aux rêves explicites, pour se couler un peu plus — il ne l'avait jamais vraiment dégradée - dans la forme classique finaliste. Mais que l'on ne s'y trompe pas : seule cette forme pourra, en éclatant l'exception du traitement des scènes de rêve (par rapport au reste de la fiction), rendre la rêverie à sa propriété modale d'être, comme je le disais à l'instant, diffuse ${ }^{60}$. Renoir, en même temps qu'il consolidera la construction aristotélicienne de ses récits (subordination du spectacle optique et sonore à la narration), ne manquera jamais de la contrecarrer, dans le même geste, par une mise en scène ouverte en permanence à toute éruption liquidatrice du travail iconique et figural $\mathrm{du}$ film (mouvements d'appareil, distraction de la prise de vues, etc.) : ce de quoi la géométrie molle de la rêverie sera donc l'un des percepts de coulure les plus cohérents et inventifs.

${ }^{59}$ J. L. Schefer, Images mobiles. Récits, visages, flocons, Paris, P.O.L, 1999, p. 144.

${ }^{60}$ G. Bachelard, La Poétique de la rêverie, op. cit., p. 144. 